\title{
Erratum
}

\section{Decays of $\tau \rightarrow \rho(770)\left(\rho^{\prime}(1450)\right) \nu_{\tau}$ and $\tau \rightarrow K^{*}(892)\left(K^{*^{\prime}}(1410)\right) \nu_{\tau}$ in the extended Nambu-Jona-Lasinio model}

[Int. J. Mod. Phys. A, Vol. 30, No. 26, 1550161 (2015)]

A. I. Ahmadov, ${ }^{*} \dagger$ Yu. L. Kalinovsky ${ }^{\ddagger}$ and M. K. Volkov*

* Bogoliubov Laboratory of Theoretical Physics, JINR, Dubna, 141980, Russia

${ }^{\dagger}$ Institute of Physics, Azerbaijan National Academy of Sciences, H. Javid ave. 131, AZ-1143 Baku, Azerbaijan

$\ddagger$ Laboratory of Information Technologies, JINR, Dubna, 141980, Russia

Published 10 April 2018

The numerical result

$$
\Gamma_{\tau \rightarrow K^{*} \nu_{\tau}}^{\mathrm{theor}}=2.60 \cdot 10^{-11} \mathrm{MeV}
$$

and

$$
\Gamma_{\tau \rightarrow K^{*^{\prime}} \nu_{\tau}}^{\text {theor }}=5.15 \cdot 10^{-12} \mathrm{MeV} .
$$

The experimental data are $\Gamma_{\tau \rightarrow K^{*} \nu_{\tau}}^{\exp }=(2.72 \pm 0.15) \cdot 10^{-11} \mathrm{MeV}, \stackrel{24}{2}$ and $\Gamma_{\tau \rightarrow K^{*^{\prime}} \nu_{\tau}}^{\exp }=$ $3.4\left(\begin{array}{c}+3.178 \\ -2.27\end{array}\right) \cdot 10^{-12} \mathrm{MeV} .24$

\section{References}

24. Particle Data Group Collab. (K. A. Olive et al.), Chin. Phys. C 38, 30 (2014). 